\title{
Comparison of clinically significant prostate cancer detection by MRI cognitive biopsy and in-bore MRI-targeted biopsy for naïve biopsy patients
}

\author{
Kai Zhang ${ }^{1,2 \#}$, Zhipeng Zhang $^{3,4 \#}$, Ming Liu ${ }^{3,4 *}$, Gang Zhu ${ }^{2 *}$, Monique J. Roobol ${ }^{1 *}$ \\ ${ }^{1}$ Department of Urology, Erasmus University Medical Center, Rotterdam, The Netherlands; ${ }^{2}$ Department of Urology, Beijing United Family \\ Hospital and Clinics, Beijing 100015, China; ${ }^{3}$ Department of Urology, Beijing Hospital, National Center of Gerontology, Beijing 100730, China; \\ ${ }^{4}$ Institute of Geriatric Medicine, Chinese Academy of Medical Sciences, Beijing 100730, China \\ Contributions: (I) Conception and design: K Zhang, MJ Roobol; (II) Administrative support: M Liu, G Zhu; (III) Provision of study materials or \\ patients: K Zhang, Z Zhang; (IV) Collection and assembly of data: K Zhang, Z Zhang; (V) Data analysis and interpretation: K Zhang, MJ Roobol; (VI) \\ Manuscript writing: All authors; (VII) Final approval of manuscript: All authors. \\ "These authors contributed equally to this work. \\ *These authors contributed equally for the senior authorship. \\ Correspondence to: Kai Zhang. Beijing United Family Hospital and Clinics, Jiangtai Road, Chaoyang District, Beijing 100015, China. \\ Email: zhangkai449@126.com.
}

Background: Multiparametric magnetic resonance imaging (mpMRI) targeted prostate biopsy increases the diagnostic accuracy of clinically significant prostate cancer (PCa). Currently there is no consensus on which type of MRI-targeted biopsy performs better in a given setting. In this study, we aimed to compare the detection rate of (clinically significant) PCa by MRI cognitive targeted biopsy (COG) and in-bore MRItargeted biopsy (IB) techniques for naïve prostate biopsy patients in China.

Methods: Our study included 85 men from Beijing United Family Hospital and Clinics and 88 men from Beijing Hospital, National Center of Gerontology. All men had no history of prostate biopsy, undergoing mpMRI scan due to elevated PSA and/or abnormal DRE. The men in Beijing United Family Hospital group received COG plus systematic biopsy. The men in Beijing Hospital group only received IB.

Results: The median age in COG and IB group was 63.0 years and 70.0 years $(\mathrm{P}<0.01)$. The median PSA was 7.4 and $6.8 \mathrm{ng} / \mathrm{mL}$ in COG and IB group respectively $(\mathrm{P}=0.124)$. The detection rate of PCa was $36.5 \%$ by $\mathrm{COG}$ and $52.3 \%$ by IB $(\mathrm{P}=0.037)$. The detection rate of clinically significant $\mathrm{PCa}(\mathrm{Gleason}$ score $\geq 7)$ was $23.5 \%$ and $29.5 \%$ by $\mathrm{COG}$ and $\mathrm{IB}(\mathrm{P}=0.371)$ respectively. In COG group, combination biopsy (COG + systematic biopsy) achieved improved PCa (42.4\%) and clinically significant PCa (28.2\%) detection rate compared with COG alone. However, there was no difference in overall PCa and clinically significant PCa detection between combination biopsy and IB.

Conclusions: IB had a higher rate of overall PCa detection compared with COG, but the two approaches did not differ significantly in the detection of clinically significant PCa. There was no significant difference in detection rate of PCa and clinically significant PCa between the combination biopsy and IB.

Keywords: Prostate cancer (PCa); magnetic resonance imaging (MRI); prostate biopsy; MRI cognitive biopsy; in-bore MRI-targeted biopsy

Submitted Nov 17, 2019. Accepted for publication Feb 14, 2020.

doi: $10.21037 /$ tau.2020.02.20

View this article at: http://dx.doi.org/10.21037/tau.2020.02.20 


\section{Introduction}

The introduction of PSA testing and subsequent transrectal ultrasound (TRUS)-guided systematic prostate biopsy led to overdiagnosis and overtreatment of $\mathrm{PCa}(1,2)$. The technique of multiparametric magnetic resonance imaging (mpMRI) can increase the sensitivity of PCa imaging and MRI-targeted biopsy improves the detection rate for clinically significant PCa (Gleason score $\geq 7$ ) as compared to the conventional TRUS-guided biopsy (3-6). MRI cognitive targeted biopsy (COG), in-bore MRI-targeted biopsy (IB) and MRI-ultrasound fusion biopsy are the three currently used techniques of MRI-targeted biopsy. There are advantages and disadvantages to each technique and it remains unknown which technique is optimal for MRItargeted biopsy (7).

Most of the studies on the performance of mpMRI and additional biopsy techniques come from Europe. The use of mpMRI and targeted biopsy was introduced in the guidelines of EAU initially only for men having to undergo a repeat biopsy. It is only recently that this changed to the recommendation to perform mpMRi in all men eligible for biopsy (8). In this study, initiated already in 2015 we present data of mpMRI and biopsy outcome from two medical centers in China using COG + systematic biopsy and the IB approach for biopsy naïve men, and compare the detection rate of $\mathrm{PCa}$ and clinically significant $\mathrm{PCa}$ between the two biopsy approaches.

\section{Methods}

\section{Study population and mpMRI protocol}

From October 2015 to May 2018, a total of 85 men from Beijing United Family Hospital and Clinics, Beijing, China and 88 men from Beijing Hospital, National Center of Gerontology, Beijing, China underwent mpMRI and subsequent MRI-targeted biopsy due to an elevated PSA $(\geq 4 \mathrm{ng} / \mathrm{mL})$ and/or abnormal DRE. All men were not previously biopsied. All men included in our study had an abnormal MRI defined as PI-RADS $\geq 2$ lesions with PCa suspicion found on MRI according to Prostate Imaging Reporting and Data System (PI-RADS) 2.0 version.

Men from Beijing United Family Hospital and Clinics received COG biopsy and additional systematic biopsy. COG was implemented using TRUS to target the suspicious lesions identified at mpMRI. A $1.5 \mathrm{~T}$ system (GE Healthcare) was used and the prostate MRI protocols included T1WI, triplanar (axial, sagittal and coronal) T2WI, diffusion weighted imaging (DWI) and dynamic contrast-enhanced imaging (DCE) by using an 8-channel phased-array coil. The operator performs 2 cores in each lesion by transperineal approach and the median number of targeted biopsy cores was 4 (IQR 2-7). The transperineal systematic biopsy was performed with a TRUS-guided approach using a median number of 12 cores (IQR 12-16).

In the Beijing Hospital group, MRIs were performed on a $3.0 \mathrm{~T}$ MR system (GE Healthcare, M750) with 16-channel pelvic phased-array coil. All the men underwent IB only, which was applied using system DynaCAD for ProstateDyna TRIM. The operator performs the transrectal biopsy and confirms the localization of needle based on the real-time MRI. Usually, 2 cores were sampled from each suspicious lesion. The median number of cores was 4 (IQR 2-6).

\section{Statistical analysis}

The Mann-Whitney test was performed to compare patient characteristics between the two biopsy approaches. The Chi-square test was used to compare the distribution of PI-RADS score and detection rate of PCa (and clinically significant $\mathrm{PCa}$, defined as Gleason $\geq 7$ ) between the two biopsy approaches. A $\mathrm{P}$ value less than 0.05 was considered statistically significant. Statistical analyses were performed by using SPSS for Windows (Version 21.0, IBM Corp Armonk, NY, USA). The sensitivity of MRI-targeted biopsy (systematic biopsy) was calculated as the number of positive MRI-targeted biopsy (systematic biopsy) results divided by the total number of cancers detected.

\section{Results}

\section{Patient characteristics}

The characteristics of the study population are shown in Table 1. In the COG group of 85 men, the median age was 63.0 years (IQR 58.5-70.0) and the median PSA value was $7.4 \mathrm{ng} / \mathrm{mL}$ (IQR 5.8-10.2). The PI-RADS score of the dominant lesion was 2 in $10(11.8 \%), 3$ in $31(36.5 \%), 4$ in $28(32.9 \%)$ and 5 in $16(18.8 \%)$ men respectively.

In the IB group of 88 men, the median age and PSA value was 70.0 (IQR 65.0-75.0) years and 6.8 (IQR 4.59.7) $\mathrm{ng} / \mathrm{mL}$ respectively. The PI-RADS was 2 in $7(8.0 \%), 3$ in $26(29.5 \%), 4$ in $47(53.4 \%)$ and 5 in $8(9.1 \%)$ men. 
Table 1 Patient characteristics

\begin{tabular}{lccc}
\hline & COG $(\mathrm{n}=85)$ & $\mathrm{IB}(\mathrm{n}=88)$ & $\mathrm{P}$ \\
\hline Age (year), median (IQR) & $63.0(58.5-70.0)$ & $70.0(65.0-75.0)$ & 0.010 \\
PSA (ng/mL), median (IQR) & $7.4(5.8-10.2)$ & $6.8(4.5-9.7)$ & 0.724 \\
PI-RADS, n (\%) & & & $7(8.0)$ \\
2 & $10(11.8)$ & $26(29.5)$ & $47(53.4)$ \\
3 & $31(36.5)$ & $8(9.1)$ & \\
4 & $28(32.9)$ & $16(18.8)$ & \\
\hline
\end{tabular}

PSA, prostate specific antigen; IQR, interquartile range; COG, MRI cognitive targeted biopsy; IB, in-bore MRI targeted biopsy; PI-RADS, Prostate Imaging Reporting and Data System.

Table 2 Detection rate of PCa and clinically significant PCa by COG and IB alone

\begin{tabular}{|c|c|c|c|}
\hline & COG ( $n=85)$, MRI-targeted biopsy & 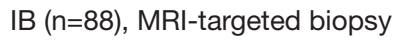 & $P$ \\
\hline $\begin{array}{l}\text { Clinically significant PCa } \\
\text { (\% of total and \% of } \mathrm{PCa} \text { ) }\end{array}$ & $20(23.5 \%, 64.5 \%)$ & $26(29.5 \%, 56.5 \%)$ & 0.371 \\
\hline
\end{tabular}

COG, MRI cognitive targeted biopsy; IB, in-bore MRI targeted biopsy; PCa, prostate cancer.

Table 3 Detection rate of PCa and clinically significant PCa by COG plus systematic biopsy and IB

\begin{tabular}{|c|c|c|c|}
\hline & COG $(n=85)$, combined biopsy & IB ( $\mathrm{n}=88)$, MRI-targeted biopsy & $\mathrm{P}$ \\
\hline $\begin{array}{l}\text { Clinically significant } \mathrm{PCa} \\
\text { (\% of total and } \% \text { of } \mathrm{PCa} \text { ) }\end{array}$ & $24(28.2 \%, 66.7 \%)$ & $26(29.5 \%, 56.5 \%)$ & 0.849 \\
\hline
\end{tabular}

COG, MRI cognitive targeted biopsy; IB, in-bore MRI targeted biopsy; PCa, prostate cancer.

\section{Detection rate of $P C a$ and clinically significant $P C a$ in the two groups}

In the COG group, COG alone detected 31 cases of PCa $(36.5 \%)$, of which $20(23.5 \%)$ cases were clinically significant $\mathrm{PCa}$. Meanwhile, IB detected significantly more cases of $\mathrm{PCa}[\mathrm{N}=46(52.3 \%), \mathrm{P}=0.037]$ but a comparable percentage of clinically significant $\mathrm{PCa}$ cases $[\mathrm{N}=26(29.5 \%)$, $\mathrm{P}=0.371$, Table 2].

In the COG group, combination biopsy (COG + systematic biopsy) detected $36(42.4 \%)$ cases of $\mathrm{PCa}$, including 24 (28.2\%) cases of clinically significant PCa. The detection rate of overall $\mathrm{PCa}$, clinically significant $\mathrm{PCa}$ was not statistically different between the COG combination biopsy and the IB (Table 3).

\section{Sensitivity of COG and Systematic biopsy in the COG group}

In the COG group, $\mathrm{COG}$ alone detected $31 \mathrm{PCa}$ of the total of $36 \mathrm{PCa}$ cases, including 20 clinically significant $\mathrm{PCa}$ of the total of 24 clinically significant PCa cases. Systematic biopsy detected 28 PCa cases including 17 clinically significant PCa cases. The sensitivity of the systematic biopsy alone was $0.78(28 / 36)$ for PCa and 0.71 (17/24) for clinically significant PCa. Compared to systematic biopsy alone, COG alone achieved superior sensitivity for both PCa $[0.86(31 / 36)]$ and clinically significant PCa $[0.83$ (20/24)] (Table 4). 
Table 4 Sensitivity of COG and Systematic biopsy in the COG group

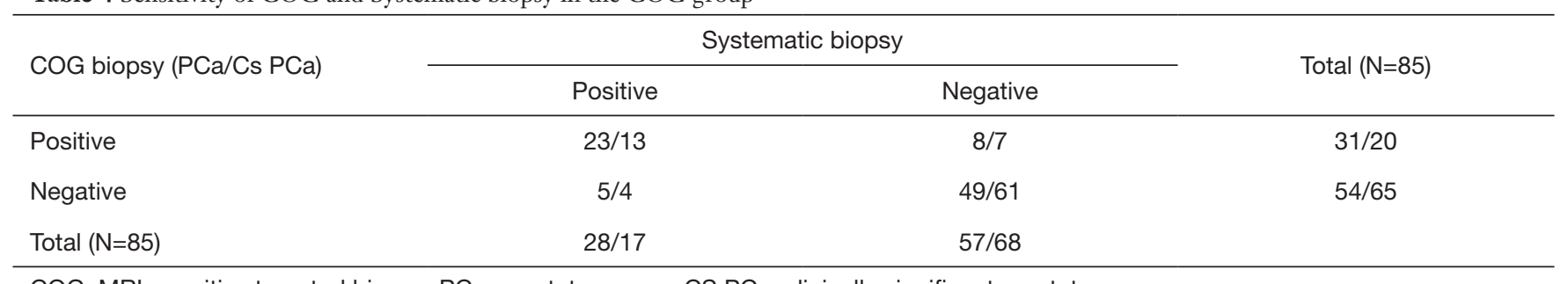

COG, MRI cognitive targeted biopsy; PCa, prostate cancer; CS PCa, clinically significant prostate cancer.

\section{Discussion}

MRI-targeted biopsy has shown great potential to improve diagnostic accuracy of clinically significant $\mathrm{PCa}$ in multiple studies (3-6,9-12). However, the detection rate of $\mathrm{PCa}$ and clinically significant PCa varies among different studies and techniques (13-15). It is still controversial which technique is preferred for MRI-targeted biopsy. Performance is most likely highly dependent on experience of both radiologist and urologist. In addition, availability of equipment (i.e., availability of resources) will also play an important role in which approach is being used.

In a systematic review and meta-analysis, 43 studies were included and the three currently used techniques were compared (16). It was shown that for overall PCa detection, there was significant advantage of using of IB compared with COG, however, for clinically significant PCa, there was no significant advantage in the performance of any one technique. In our study, for overall PCa, IB showed a significantly higher detection rate as compared to COG. For clinically significant PCa detection, COG and IB did not differ significantly, making our findings consistent with the meta-analysis (16).

In an Australian study, 482 men with PI-RADS 3-5 lesions were included with the aim to compare IB with COG (transrectal and transperineal) (17). The study showed that there was no significant difference in $\mathrm{PCa}$ detection among IB, transperineal COG and transrectal COG in PI-RADS 3 (48.9\%, 40.0\%, 44.4\%), PI-RADS 4 (73.2\%, $81.0 \%, 85.0 \%)$ or PI-RADS 5 (95.2, 92.0\%, 95.0\%) lesions. For clinically significant $\mathrm{PCa}$, the detection rate also did not differ between the three techniques in PI-RADS 3 (42.2\%, $30.0 \%, 33.3 \%)$, PI-RADS $4(66.8 \%, 66.0 \%, 80.0 \%)$ or PIRADS 5 (90.5\%, 89.8\%, 90.0\%) lesions.

As is known, one of the main concerns for IB is the cost of the procedure. It is still a relatively new technique, requiring special MR compatible equipment and demagnetization of the biopsy gun and related instruments resulting in extra costs. In a Dutch study, the cost-effectiveness was compared among TRUS systematic biopsy, IB and MRI-Ultrasound fusion biopsy. It showed that MRI-TRUS fusion biopsy is cost-effective compared with TRUS systematic biopsy. However, only if the sensitivity of IB for clinically significant PCa is at least $89 \%$, IB would be the most cost-effective strategy (18). In our study, the sensitivity for clinically significant PCa was $83 \%$ by IB, and one could question cost effectiveness. With the current available data on the additional value of an IB approach, the requirements for successful implementation of the IB technique, especially for those regions with limited health care expenditures, is unlikely.

Another point of concern with the IB approach is that the procedure is relatively time consuming. One study reported that the mean duration was $55 \mathrm{~min}$ (19). Generally, during the procedure a systematic biopsy is not performed mainly due to the time and expense required for each biopsy in the MRI magnet environment (20).

Compared to MRI targeted biopsies, the systematic biopsy has been shown to increase cancer detection rates (21). Also, in the COG group of our study, if only COG would have been performed, $5(13.9 \%, 5 / 36)$ cases of PCa including 4 (16.7\%, 4/24) clinically significant PCa would have been missed. In contrast, if only systematic biopsy was performed, $8(22.2 \%, 8 / 36)$ cases of $\mathrm{PCa}$ would have been missed and $7(29.2 \%, 7 / 24)$ of those were clinically significant PCa.

This complementary effect of the two biopsy approaches is confirmed by data from a French study of 555 men with suspicion of $\mathrm{PCa}$, where all the men underwent 10-12 cores systematic biopsy plus two cores COG. Among 151 men with positive MRI, combination biopsy detected $71.8 \%$ tumors (252/351), which was higher than COG (67.2\%, $236 / 351)$ or systematic biopsy alone $(68.4 \%, 240 / 351)$ alone (22). A Japanese study also showed that systematic biopsy missed $34.6 \%$ of PCa (18 of 52) compared with 
$13.5 \%$ (7 of 52) for the MRI-targeted biopsy (23). A recently published meta-analysis indicated that MRItargeted biopsy combined with systematic biopsy maximized PCa detection as compared to MRI-targeted biopsy or systematic biopsy alone in men on active surveillance (24).

It is interesting to note that in one study the shape and size of suspicious lesion on mpMRI was compared with the specimen of localized PCa after radical prostatectomy. It showed that the mean pathological tumour volume was three-times greater than the mean region of interest volume indicated on mpMRI $(\mathrm{P}<0.001)$ and the tumour diameter was significantly underestimated by an average of $11 \mathrm{~mm}$, with the tumour extending beyond the region of interest on all anatomical axes (25). The authors suggested that underestimation of tumour volume may be compensated for during the biopsy process by taking additional cores outside each suspicious lesion, which indicated that only performing MRI-targeted biopsy may not adequate enough. Another study revealed that lower MRI lesion volumes, lesion density, and PI-RADS score were significantly associated with PCa detected by systematic biopsy but missed by targeted biopsy (26).

The European Association of Urology revised the guidelines in 2019, advised to perform a MRI scan even for initial biopsy patient and to provide subsequent MRItargeted biopsy and systematic biopsy for PI-RADS $\geq 3$ lesions. It is interesting to note that in our study, IB alone achieved mildly higher no statistically significant PCa and clinically significant $\mathrm{PCa}$ detection rate than combination biopsy. In a recently published multicenter, randomized study, 500 naïve biopsy men with suspicion of PCa were assigned to undergo MRI, with or without targeted biopsy, or transrectal systematic biopsy. It demonstrated that the clinically significant PCa detection rate was 38\% in MRItargeted biopsy group and $26 \%$ in systematic biopsy group $(\mathrm{P}=0.005)$ (27). This is the first study indicating that next to MRI targeted biopsy, systematic biopsy might not be necessary. Until these data are confirmed in additional studies omitting systematic biopsy remains controversial.

The strengths of our study include the fact that the two cohorts were closely matched in demographics and disease characteristics. There were no significant differences between the two group in terms of PSA level and distribution of PI-RADS score, and all men in this study were not previously biopsied. In addition, all patients were sampled from two tertiary hospitals with high skills in prostate MRI image acquisition and interpretation. In addition, the urologists had ample experience in systematic and MRI-targeted biopsy.

Our study is limited by its retrospective nature. The patients came from two institutions with different MR equipment and the MRI images were reviewed by different radiologists. Additionally, the two groups differed in median age even these are consecutive patients. However, there were no differences in PSA level and PI-RADS score between groups. Second, the sample size was relatively small in this study, 85 and 88 men were included in the two cohorts. Third, our follow-up is limited after prostate biopsy. Data from those patients referred to repeat biopsy remains absent and the final pathology results from radical prostatectomy specimens are still limited. Furthermore, in some patients, bias may have been caused by the relatively low number of MRI- targeted biopsy cores for the sampled PCa volume.

\section{Conclusions}

In this Asian population where also men scheduled for initial biopsy underwent mpMRi and targeted biopsy, there were no significant difference in the detection rate of $\mathrm{PCa}$ and clinically significant $\mathrm{PCa}$ between the combination biopsy approach of COG fusion biopsy including systematic biopsies and an IB biopsy approach only. A combination biopsy might be more cost-effective in our setting since there is no need for special equipment using the IB biopsy approach. The sensitivity of the combination biopsy for the detection of PCa and clinically significant PCa was superior as compared to a systematic biopsy approach only.

\section{Acknowledgments}

Funding: None.

\section{Footnote}

Conflicts of Interest: All authors have completed the ICMJE uniform disclosure form (available at http://dx.doi. org/10.21037/tau.2020.02.20). The authors have no conflicts of interest to declare.

Ethical Statement: The authors are accountable for all aspects of the work in ensuring that questions related to the accuracy or integrity of any part of the work are appropriately investigated and resolved. The International Review Board (IRB) of the Beijing United Family Hospital 
and Clinics (Medical Ethical Committee) approved our database/study (UFHIRB.E 2018-0004).

Open Access Statement: This is an Open Access article distributed in accordance with the Creative Commons Attribution-NonCommercial-NoDerivs 4.0 International License (CC BY-NC-ND 4.0), which permits the noncommercial replication and distribution of the article with the strict proviso that no changes or edits are made and the original work is properly cited (including links to both the formal publication through the relevant DOI and the license). See: https://creativecommons.org/licenses/by-ncnd/4.0/.

\section{References}

1. Schröder FH, Hugosson J, Roobol MJ, et al. Screening and prostate-cancer mortality in a randomized European study. N Engl J Med 2009;360:1320-8.

2. Schröder FH, Hugosson J, Roobol MJ, et al. Prostatecancer mortality at 11 years of follow-up. N Engl J Med 2012;366:981-90.

3. Siddiqui MM, Rais-Bahrami S, Turkbey B, et al. Comparison of MR/ultrasound fusion-guided biopsy with ultrasound-guided biopsy for the diagnosis of prostate cancer. JAMA 2015;313:390-7.

4. Schoots IG, Roobol MJ, Nieboer D, et al. Magnetic resonance imaging-targeted biopsy may enhance the diagnostic accuracy of significant prostate cancer detection compared to standard transrectal ultrasound-guided biopsy: a systematic review and meta-analysis. Eur Urol 2015;68:438-50.

5. NiMhurchu E, O'Kelly F, Murphy IG, et al. Predictive value of PI-RADS classification in MRI-directed transrectal ultrasound guided prostate biopsy. Clin Radiol 2016;71:375-80.

6. Pokorny MR, de Rooij M, Duncan E, et al. Prospective study of diagnostic accuracy comparing prostate cancer detection by transrectal ultrasound-guided biopsy versus magnetic resonance (MR) imaging with subsequent MRguided biopsy in men without previous prostate biopsies. Eur Urol 2014;66:22-9.

7. Giganti F, Moore CM. A critical comparison of techniques for MRI-targeted biopsy of the prostate. Transl Androl Urol 2017;6:432-43.

8. EAU Guidelines: Prostate Cancer 2019. Available online: https://uroweb.org/guideline/prostatecancer/?type=summary-of-changes
9. Portalez D, Mozer P, Cornud F, et al. Validation of the European Society of Urogenital Radiology scoring system for prostate cancer diagnosis on multiparametric magnetic resonance imaging in a cohort of repeat biopsy patients. Eur Urol 2012;62:986-96.

10. Fütterer JJ, Briganti A, De Visschere P, et al. Can Clinically Significant Prostate Cancer Be Detected with Multiparametric Magnetic Resonance Imaging? A Systematic Review of the Literature. Eur Urol 2015;68:1045-53.

11. Nam RK, Wallis CJ, Stojcic-Bendavid J, et al. A Pilot Study to Evaluate the Role of Magnetic Resonance Imaging for Prostate Cancer Screening in the General Population. J Urol 2016;196:361-6.

12. Meng X, Rosenkrantz AB, Mendhiratta N, et al. Relationship Between Prebiopsy Multiparametric Magnetic Resonance Imaging (MRI), Biopsy Indication, and MRI-ultrasound Fusion-targeted Prostate Biopsy Outcomes. Eur Urol 2016;69:512-7.

13. Wysock JS, Rosenkrantz AB, Huang WC, et al. A prospective, blinded comparison of magnetic resonance (MR) imaging-ultrasound fusion and visual estimation in the performance of MR-targeted prostate biopsy: the PROFUS trial. Eur Urol 2014;66:343-51.

14. Kasivisvanathan V, Dufour R, Moore CM, et al. Transperineal magnetic resonance image targeted prostate biopsy versus transperineal template prostate biopsy in the detection of clinically significant prostate cancer. J Urol 2013;189:860-6.

15. Quentin M, Schimmoller L, Arsov C, et al. 3-T inbore MR-guided prostate biopsy based on a scoring system for target lesions characterization. Acta Radiol 2013;54:1224-9.

16. Wegelin O, van Melick HH, Hooft L, et al. Comparing Three Different Techniques for Magnetic Resonance Imaging-targeted Prostate Biopsies: A Systematic Review of In-bore versus Magnetic Resonance Imaging-transrectal Ultrasound fusion versus Cognitive Registration. Is There a Preferred Technique? Eur Urol 2017;71:517-31.

17. Yaxley AJ, Yaxley JW, Thangasamy IA, et al. Comparison between target magnetic resonance imaging (MRI) in-gantry and cognitively directed transperineal or transrectal-guided prostate biopsies for Prostate ImagingReporting and Data System (PI-RADS) 3-5 MRI lesions. BJU Int 2017;120 Suppl 3:43-50.

18. Venderink W, Govers TM, de Rooij M, et al. CostEffectiveness Comparison of Imaging-Guided Prostate Biopsy Techniques: Systematic Transrectal Ultrasound, 
Direct In-Bore MRI, and Image Fusion. AJR Am J

Roentgenol 2017;208:1058-63.

19. Felker ER, Lee-Felker SA, Feller J, et al. In-bore magnetic resonance-guided transrectal biopsy for the detection of clinically significant prostate cancer. Abdom Radiol (NY) 2016;41:954-62.

20. Robertson NL, Emberton M, Moore CM. MRI-targeted prostate biopsy: a review of technique and results. Nat Rev Urol 2013;10:589-97.

21. Ploussard G, Borgmann H, Briganti A, et al. Positive prebiopsy MRI: are systematic biopsies still useful in addition to targeted biopsies? World J Urol 2019;37:243-51.

22. Haffner J, Lemaitre L, Puech P, et al. Role of magnetic resonance imaging before initial biopsy: comparison of magnetic resonance imaging-targeted and systematic biopsy for significant prostate cancer detection. BJU Int 2011;108:E171-8.

23. Miyagawa T, Ishikawa S, Kimura T, et al. Real-time Virtual Sonography for navigation during targeted prostate biopsy using magnetic resonance imaging data. Int J Urol 2010;17:855-60.

24. Schoots IG, Nieboer D, Giganti F, et al. Is magnetic resonance imaging-targeted biopsy a useful addition to systematic confirmatory biopsy in men on active surveillance for low-risk prostate cancer? A systematic review and meta-analysis. BJU Int 2018;122:946-58.

25. Priester A, Natarajan S, Khoshnoodi P, et al. Magnetic Resonance Imaging Underestimation of Prostate Cancer Geometry: Use of Patient Specific Molds to Correlate Images with Whole Mount Pathology. J Urol 2017;197:320-6.

26. Coker MA, Glaser ZA, Gordetsky JB, et al. Targets missed: predictors of MRI-targeted biopsy failing to accurately localize prostate cancer found on systematic biopsy. Prostate Cancer Prostatic Dis 2018;21:549-55.

27. Kasivisvanathan V, Rannikko AS, Borghi M, et al. MRITargeted or Standard Biopsy for Prostate-Cancer Diagnosis. N Engl J Med 2018;378:1767-77.

Cite this article as: Zhang K, Zhang Z, Liu M, Zhu G, Roobol MJ. Comparison of clinically significant prostate cancer detection by MRI cognitive biopsy and in-bore MRItargeted biopsy for naïve biopsy patients. Transl Androl Urol 2020;9(2):243-249. doi: 10.21037/tau.2020.02.20 